\title{
Probing Quasiparticle States Bound by Disparate Periodic Potentials
}

\author{
P. Moras, ${ }^{1}$ L. Ferrari, ${ }^{1,2}$ C. Spezzani, ${ }^{1}$ S. Gardonio, ${ }^{1}$ M. Ležaić, ${ }^{3}$ Ph. Mavropoulos, ${ }^{3}$ S. Blügel, ${ }^{3}$ and C. Carbone ${ }^{1}$ \\ ${ }^{1}$ Istituto di Struttura della Materia, Consiglio Nazionale delle Ricerche, Trieste, Italy \\ ${ }^{2}$ Istituto dei Sistemi Complessi, Consiglio Nazionale delle Ricerche, Roma, Italy \\ ${ }^{3}$ Institut für Festkörperforschung, Forschungszentrum Jülich, D-52425 Jülich, Germany
}

(Received 23 May 2006; published 13 November 2006)

\begin{abstract}
Thin films of $\operatorname{Ag}(111)$ with two-dimensional crystallinity of large lateral coherence grow on $\mathrm{Ge}(111)$, free of in-plane registry with the underlying substrate. Ag $s$ - $p$ electrons forming two-dimensional quantum well states scatter coherently at the buried interface potential, resulting in an unexpected set of new quasiparticle states, as observed by angle-resolved photoemission. These new features originate from interactions among Ag quantum well bands, gaining a momentum equivalent to a reciprocal vector of the substrate lattice.
\end{abstract}

DOI: 10.1103/PhysRevLett.97.206802

Translational symmetry is an essential property in crystals, which gave birth to fundamental concepts such as periodic potentials, crystal momentum, Brillouin zones, and electronic bands. Breaking this symmetry challenges our understanding of the electronic structure and opens new perspectives in modifying the electronic, magnetic, growth, and transport properties as well as low-temperature phenomena such as the superconductivity and the Kondo physics. In a self-standing layer with a thickness of the order of the de Broglie wavelength, the breaking of the translational symmetry along the direction normal to the surface planes leads to a quantization of the energy levels. Similarly, in a thin metal film on a substrate the interface potential can act as a reflecting wall on the electron wave functions, giving rise to two-dimensional (2D) quantum well (QW) states, partially or completely confined within the film [1]. The degree of localization of QW states inside the film depends on their hybridization with the substrate bands, which is defined by the overlap of the states in the two materials with corresponding energy and symmetry. Because of the boundary conditions, the properties of QW states reflect thus in detail the electronic interactions at the interface with the supporting substrate [2-6].

A much more complex scenario arises when the translational symmetry is broken such that electrons reside in potentials with disparate translational periodicities. For instance, competing electron-electron or electron-phonon interactions drive particularly low-dimensional systems into charge- or spin-density wave states [7-12], which are incommensurable with the underlying crystal lattice. Alternatively, in artificially ordered 2D heterojunctions [13] or tunnel junctions [14], relevant for nanoelectronics and spintronics, electrons are coherently transported between two structures of different periodicities. Besides being of practical interest, these systems raise attractive and unresolved fundamental questions on how their electronic structure depends on the translational mismatch, the difference in the potential strength between the crystalline structure of the two constituents, and the dissimilarity of the electronic structure.
PACS numbers: 73.21.Fg, 73.22.Gk, 73.40.Ns

We notice that recently, several photoemission experiments focused on QW states of noble and simple metal layers on semiconductors, aiming at the understanding of coupling effects among film and substrate electronic states [15-17]. The difference in the lattice parameters between metals and semiconductors does not necessarily hinder a single-crystal film growth but often results in very large unit cells or incommensurate structures. Moreover, the difference in the point group symmetry of these metals and the diamond or zinc-blende semiconductors implies that the interaction between bands with common symmetry has to be limited to particular regions in the momentumenergy space. QW states display in several systems an enhancement of the effective electron mass arising from the hybridization between substrate and metal states $[15,16]$. The interactions with the substrate most remarkably result in discontinuities of the QW dispersionobservable, also in relatively thick films, when the QW states cross the upper edge of the semiconductor valence band and enter into a $k$-projected energy gap $[16,17]$. Although a detailed understanding of these effects on the basis of first-principle band structure calculations has until now not been attempted, all these observations could be directly related to the interaction between the electronic states of the film and the bulk bands of the semiconductor substrate.

Here we show that the electronic structure of a metal film on a semiconductor can be modulated by interface interactions, which break the in-plane translational symmetry and do not involve the hybridization with the substrate bands. In order to shed light onto the behavior of electrons in a multiperiodic potential, we examined by angle-resolved photoemission the QW states of thin $\mathrm{Ag}$ films grown on a $\mathrm{Ge}(111)$ surface. The films form an incommensurate interface, due to the absence of simple matching relations with the substrate lattice parameters. We demonstrate that the whole electronic structure of the metal films conforms to the incommensurate potential developed at the buried interface and acquires clear signatures of the substrate lattice periodicity. 
Angle-resolved photoemission measurements were carried out on the VUV-beam line at the Elettra synchrotron radiation source in Trieste. The surface of $n$-type Ge(111) wafers was prepared by repeated cycles of $\mathrm{Ar}^{+}$sputtering and resistive annealing to display a $c(2 \times 8)$ reconstruction. Ag films were deposited by evaporation from a resistive crucible source, which was calibrated by using a quartz microbalance. The samples were held at $140 \mathrm{~K}$ during $\mathrm{Ag}$ deposition and then slowly annealed to room temperature to favor a layer-by-layer growth, with formation of a sharp interface [18]. For film thickness above four monolayers (ML), the low energy electron diffraction reveals a (111) pattern with the same in-plane orientation of the substrate. The lattice constant of the film structure, measured by comparing the distance between $(0,0)$ and $(1,0)$ diffraction spots of $\mathrm{Ag}$ films and bare $\mathrm{Ge}(111)$ surface, is determined to be very close to the $\mathrm{Ag}(111)$ bulk truncated case $\left(2.52 \pm 0.08 \AA^{-1}\right)$. Photoemission spectra were measured with linearly polarized radiation, incident on the sample at an angle of $45^{\circ}$ from the surface normal. The electron analyzer was operated with a full-cone angular acceptance of $1^{\circ}$. The data here presented are obtained with photon energy of $47 \mathrm{eV}$, which optimizes the photoemission signal from the QW states. Photoemission spectra were measured for several other photon energies in order to assess the quasi-2D character of these states.

Photoemission spectra measured along the $\bar{\Gamma}-\bar{M}$ axis for an $8 \mathrm{ML} \mathrm{Ag} \mathrm{film} \mathrm{are} \mathrm{reported} \mathrm{in} \mathrm{Fig.} \mathrm{1.} \mathrm{The} \mathrm{most} \mathrm{intense}$ and sharp peak, just below the Fermi level in near-normal emission geometry (thick line), is identified as the $\operatorname{Ag}(111)$ Shockley surface state. Two QW states are detected for this film thickness, at binding energies within $3.5 \mathrm{eV}$ from the Fermi level. These states arise from the confinement of the Ag $s, p$ valence electrons within the metal film. Their dispersion as a function of the in-plane wave vector displays small discontinuities at points that correspond to the crossings of the Ge band edges projected on the (111) plane, in close correspondence with the observation reported in a previous study for the $\bar{\Gamma}-\bar{K}$ direction [17].

Moreover, additional features appear in the spectra in off-normal geometry. In the region around $15^{\circ}$ emission, narrow peaks exhibit a flat in-plane dispersion, as indicated by the continuous lines in Fig. 1. Similarly, these features are again observed around $28^{\circ}$ emission angle, although with reduced intensity. Figure 2 reports in a color-scale map the photoemission data of Fig. 1, along with the bulk band structure of Ge projected on the (111) plane [19]. It is important to notice that the shallow bands fall in the fundamental gap of Ge, thus indicating that they purely originate from the valence states of the metal layer. Additional measurements on thicker films (not shown here) demonstrate that the number and the energy separation of the bands depend on the Ag film thickness, alike to the QW parabolas that originate at $\bar{\Gamma}$. Most remarkably, the wave vector corresponding to these features appears to be directly related to the substrate lattice parameter along the

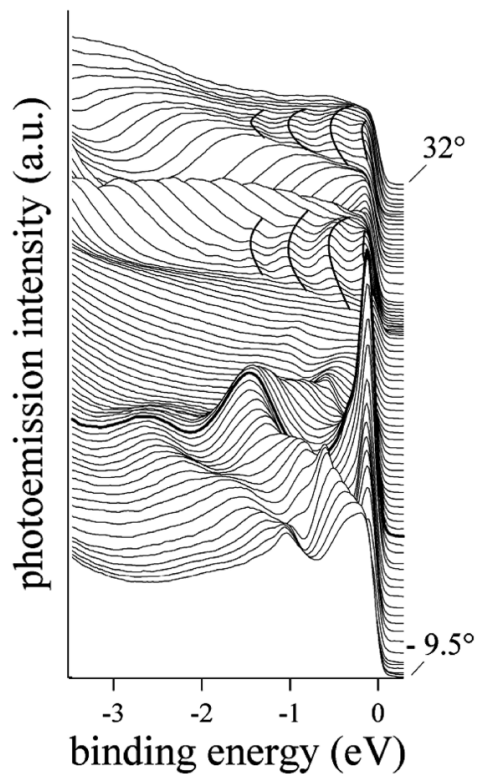

FIG. 1. Set of photoemission spectra recorded along the $\bar{\Gamma}-\bar{M}$ axis for an $8 \mathrm{ML} \mathrm{Ag} \mathrm{film.} \mathrm{Continuous} \mathrm{lines} \mathrm{guide} \mathrm{the} \mathrm{eye} \mathrm{through}$ the in-plane dispersion of the shallow bands.

$\bar{\Gamma}-\bar{M}$ direction. The features centered around $15^{\circ}$ emission exhibit a parabolic dispersion with minima precisely located at the reciprocal vector corresponding to the $\bar{M}$ point of the surface Brillouin zone of $\mathrm{Ge}\left(\bar{M}_{\mathrm{Ge}}=0.91 \AA^{-1}\right)$. Symmetrically, with respect to the zone boundary of the Ag film $\left(\bar{M}_{\mathrm{Ag}}=1.25 \AA^{-1}\right)$, the second series of shallow bands falls at $1.59 \AA^{-1}$, the location of $\bar{M}_{\mathrm{Ge}}$ referred to the center of next zone of $\mathrm{Ag}\left(\bar{\Gamma}_{\mathrm{Ag}}^{\prime}\right)$. These simple relations suggest that the novel electronic features observed in the QW bands reflect the properties of the buried interface formed by the two incommensurate material lattices.

These experimental findings can be explained employing a model film of thickness $d$, with $x$ and $y$ in-plane axes and $z$ normal to the surface. For clarity, the following dis-

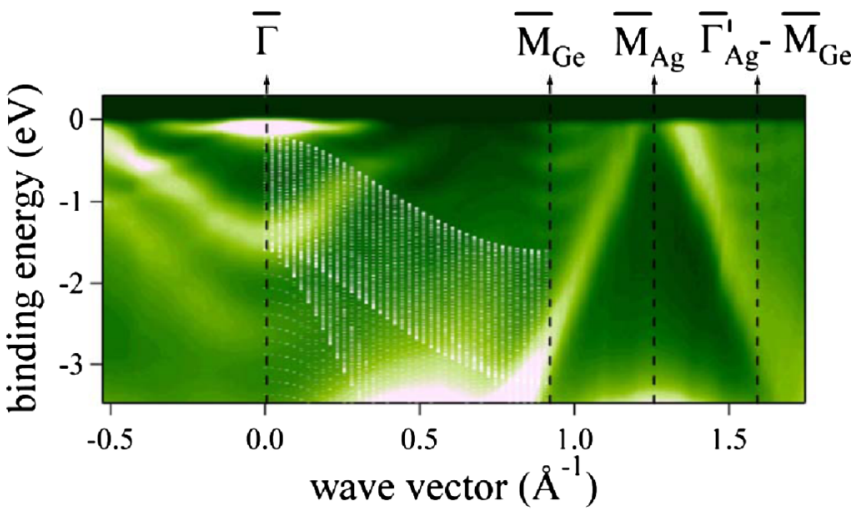

FIG. 2 (color online). Photoemission intensity plot obtained from the data of Fig. 1. The positions of the film and substrateinduced Bragg planes are marked. White dots represent the (111)-projected bulk band structure of Ge. 
cussion considers only one in-plane direction $(x)$. The potential $V_{A}(x, z)$, acting on all electrons of the system, is periodic along $x$, with periodicity $a_{A}$, and confining along $z$, and is assumed to be weak, a realistic ansatz for the weakly scattering $s$ electrons of Ag. The confinement along $z$ results in a series of mutually orthogonal QW states, labeled by the number of nodes $l$, having the form $\psi_{l k}(x, z)=\varphi_{k}(x) \chi_{l}(z)$, with $\varphi_{k}(x)$ obeying Bloch's theorem and $\chi_{l}(z)=\sin [(l+1) \pi z / d]$, if $V_{A}$ is constant in the $z$ direction for $0 \leq z \leq d$. In an extended-zone scheme, the periodic potential induces energy gaps at the Bragg planes located at $(1 / 2) G_{A}^{n}$, where $G_{A}^{n}=2 \pi n / a_{A}$ and $n=$ $0, \pm 1, \pm 2 \ldots$ They can be viewed as hybridization gaps between bands of the same $l$ centered at various $G_{A}^{n}$.

If two potentials $V_{A}$ and $V_{B}$, with commensurate inplane periods $a_{A}$ and $a_{B}$, respectively, act simultaneously in the model film, the total potential $V=V_{A}+V_{B}$ will have a supercell in-plane periodicity $\tilde{a} \geq \max \left\{a_{A}, a_{B}\right\}$. The original bands corresponding to $V_{A}$ are now backfolded into the new reduced Brillouin zone and interact due to the new potential $V_{B}$. The Fourier components of the composite potential have nonzero values only for those reciprocal vectors belonging to either sublattice, $G_{A}^{n}=2 \pi n / a_{A}$ or $G_{B}^{m}=2 \pi m / a_{B}$. Now, gaps open at $(1 / 2) G_{A}^{n}$ (as previously) and, additionally, at $G_{A}^{n} \pm(1 / 2) G_{B}^{m}$, by hybridization of bands centered at $G_{A}^{n}$ with backfolded bands centered at $G_{A}^{n} \pm G_{B}^{m}$. Again, only bands of the same $l$ hybridize: states of different numbers of nodes are still orthogonal. The incommensurate case is obtained in the limit of large or infinite supercells [8,9].

In the presented experiment $V_{A}$ corresponds to the bare Ag potential and $V_{B}$ to the perturbation introduced by the $\mathrm{Ge}$ substrate. Notably, this perturbation acts on the $\mathrm{Ag}$ wave functions only at the interface: therefore $V_{B}$ is localized only in a part of the film, close to the plane $z=0$. Because of this limitation, original and backfolded subbands of different $l$ can hybridize and open gaps at and close to $G_{A}^{n} \pm(1 / 2) G_{B}^{m}$. Intuitively, the coupling between subbands of different numbers of nodes $l \neq j$ arises because $V_{B}$ introduces at the interface different phase shifts to the wave functions $\chi_{l}(z)$ and $\chi_{j}(z)$, due to the different kinetic energy associated with the motion along $z$.

As an example of the previous analysis, a $2 \mathrm{D}$ singleorbital tight-binding model with exponentially decreasing hopping is considered. Although too simplistic to realistically reproduce the details of the band structure of the $\mathrm{Ag} / \mathrm{Ge}$ system, this model is adequate to show the effect. Figure 3(a) shows the band structure for a 4-layer case of in-plane lattice constant $a_{A}$. The spectral weight of the bands, proportional to the photoemission signal $[8,20]$, is related to the line thickness. Also backfolded ("shadow") bands, corresponding to a doubling of the unit cell, are drawn in gray - in this case, the shadow bands have no spectral weight. If a weak perturbing potential $V_{B}$, with periodicity $a_{B}=2 a_{A}$ and acting equally on all layers, is added, gaps are formed at $( \pm 1 / 4) \pi / a_{A}$ due to hybridiza-
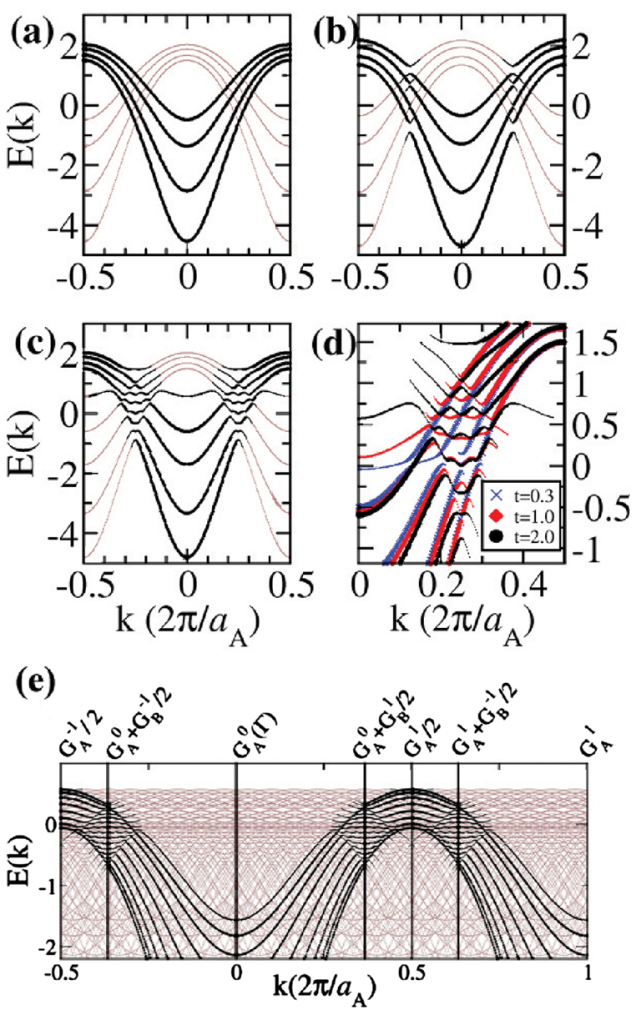

FIG. 3 (color online). Band structure resulting from a 2D tightbinding model of a film. The spectral weight of the bands, proportional to the photoemission signal $[8,20]$, is related to the line thickness in the plots. (a) Four layers in a potential $V_{A}$ with periodicity $a_{A}$. (b) Same as in (a), with an additional potential $V_{B}$ with periodicity $a_{B}=2 a_{A}$, acting on all layers. (c) Same as in (a), with an additional substrate layer of lattice constant $a_{B}=2 a_{A}$. QW states of the new type appear. (d) Modulation of the new type of QW states as the hopping parameter to and in the interface scales by a factor $t ; t=1$ corresponds to the same hopping as within the unperturbed film. (e) Band structure resulting from a 2D tight-binding model of a film of 8 layers and a periodic modulation at the interface with a film/substrate lattice constant ratio $a_{A} / a_{B}=11 / 15$.

tion of original with shadow subbands of the same $l$ [Fig. 3(b)]. The shadow bands obtain some spectral weight close to these gaps, becoming visible by photoemission. Finally, if $V_{B}$ is limited to an additional substrate layer of in-plane lattice constant $a_{B}=2 a_{A}$, simulating the effect of an interface, subbands of different $l$ do hybridize and give rise to new band patterns with "zigzag" dispersion [Fig. 3(c)]. When the potential strength increases, larger gaps form. If they assume a value comparable to the energy separation between successive subbands, the resulting band dispersion will then follow a smoothly interpolated behavior. Figure 3(d) shows the change of the zigzag pattern as the coupling strength $t$ (hopping element) to the substrate is changed: higher $t$ leads to the formation of larger gaps.

This analysis remains valid for any ratio $a_{A} / a_{B}$. Figure 3(e) shows the result for a 8-layer film with $a_{A} / a_{B}=11 / 15$, approximating the ratio between $\mathrm{Ag}$ and 
Ge lattice constants. Evidently, gaps and new band patterns are formed around $\pm(1 / 2) G_{B}^{1}= \pm(11 / 15) \pi / a_{A}, G_{A}^{1}+$ $(1 / 2) G_{B}^{-1}=(19 / 15) \pi / a_{A}$, etc., induced by the hybridization of original QW states departing from $k_{\|}=0$ with shadow QW states departing from $2 \pi / a_{B}$, i.e., from the first reciprocal lattice vector corresponding to the perturbing potential $V_{B}$. We conclude that the states of the new type come about from the spatial confinement of $V_{B}$ at the interface, allowing for the hybridization of subbands with different number of nodes.

Crucial for the detection of the interaction among original and shadow states in a photoemission experiment is the intensity of the spectral function, which depends on the strength of the scattering potential [20,21], the lateral coherence of the $\mathrm{Ag} / \mathrm{Ge}$ interface, and the absence of $\mathrm{Ge}$ bulk projected bands (Fig. 2) in order to avoid any confusion by hybridization with the newly formed 2D states.

The origin of the novel structures discovered in the QW dispersion can be identified with the umklapp processes initiated at the buried interface, occurring through exchange of the substrate reciprocal vectors. In real space, these vectors are directly associated with the modulation of the electronic potential felt by the $\mathrm{Ag}$ electrons at the interface, and, possibly, to a corresponding atomic buckling induced in the interface layers of the $\mathrm{Ag}$ film. The $\bar{\Gamma}-\bar{M}$ direction is particularly favorable for the observation of the band crossing because of its high symmetry character. Nevertheless, band crossings can be expected at other points in reciprocal space, where QW states originating from various Brillouin zones can interact. Moreover, it can be anticipated that the umklapp processes here reported are likely to be generally relevant in a broad class of lattice mismatched film-substrate systems, although their observation in photoemission experiments may be difficult in materials with more complex electronic structure.

From photoemission data obtained on thicker films it is clear that the new states display the same behavior of the QW states centered at the $\bar{\Gamma}$ point: their energy separation, as well as their intensity, decreases monotonically with increasing film thickness. We expect such behavior, since our analysis demonstrates that the new quasiparticle states derive from replicas of the original QW states, translated by reciprocal vectors derived from the Ge lattice periodicity.

In conclusion, by means of angle-resolved photoemission, we investigated the effects of disparate periodic lattices on the electronic properties of thin metallic films. We have shown that QW states of $\mathrm{Ag}(111)$ films on $\mathrm{Ge}$ develop a characteristic band structure as a consequence of their coupling with the semiconductor lattice. In particular, the QW states in this system provide the first direct evidence for the relevance of umklapp processes at a buried interface, mediated by the incommensurate lattice vectors. This work opens a new perspective of tuning electronic properties of low-dimensional materials by exploring system combinations with larger scattering strength.
Note added in proof.-We noticed that after the submission of this manuscript another Letter on the same subject has been published [22].

[1] F. J. Himpsel, J. E. Ortega, G. J. Mankey, and R. F. Willis, Adv. Phys. 47, 511 (1998); T.-C. Chiang, Surf. Sci. Rep. 39, 181 (2000).

[2] K. Garrison, Y. Chang, and P. D. Johnson, Phys. Rev. Lett. 71, 2801 (1993); C. Carbone, E. Vescovo, O. Rader, W. Gudat, and W. Eberhardt, Phys. Rev. Lett. 71, 2805 (1993).

[3] R. K. Kawakami, E. Rotenberg, Hyuk J. Choi, Ernesto J. Escorcia-Aparacio, M. O. Bowen, J.H. Wolfe, E. Arenholz, Z. D. Zhang, N. V. Smith, and Z. Q. Qiu, Nature (London) 398, 132 (1999).

[4] J. J. Paggel, T. Miller, and T.-C. Chiang, Science 283, 1709 (1999).

[5] C. Koitzsch, C. Battaglia, F. Clerc, L. Despont, M. G. Garnier, and P. Aebi, Phys. Rev. Lett. 95, 126401 (2005).

[6] A. Varykhalov, A. M. Shikin, W. Gudat, P. Moras, C. Grazioli, C. Carbone, and O. Rader, Phys. Rev. Lett. 95, 247601 (2005).

[7] G. Grüner, Density Waves in Solids (Addison-Wesley, Reading, MA, 1994).

[8] J. Voit, L. Perfetti, F. Zwick, H. Berger, G. Margaritondo, G. Grüner, H. Höchst, and M. Grioni, Science 290, 501 (2000).

[9] J. Schäfer, Eli Rotenberg, S. D. Kevan, P. Blaha, R. Claessen, and R. E. Thorne, Phys. Rev. Lett. 87, 196403 (2001).

[10] F. Baumberger, A. Tamai, M. Muntwiler, T. Greber, and J. Osterwalder, Surf. Sci. 532-535, 82 (2003).

[11] V. Brouet, W. L. Yang, X. J. Zhou, Z. Hussain, N. Ru, K. Y. Shin, I. R. Fisher, and Z.X. Shen, Phys. Rev. Lett. 93, 126405 (2004).

[12] F. Schiller, J. Cordón, D. Vyalikh, A Rubio, and J.E. Ortega, Phys. Rev. Lett. 94, 016103 (2005).

[13] P. Bruno and C. Chappert, Phys. Rev. Lett. 67, 1602 (1991); J.E. Ortega and F. J. Himpsel, Phys. Rev. Lett. 69, 844 (1992).

[14] Ph. Mavropoulos, N. Papanikolaou, and P. H. Dederichs, Phys. Rev. Lett. 85, 1088 (2000); E. Y. Tsymbal, O. N. Mryasov, and P. R. LeClair, J. Phys. Condens. Matter 15, R109 (2003).

[15] L. Aballe, C. Rogero, P. Kratzer, S. Gokhale, and K. Horn, Phys. Rev. Lett. 87, 156801 (2001).

[16] I. Matsuda, T. Ohta, and H. W. Yeom, Phys. Rev. B 65, 085327 (2002).

[17] S.-J. Tang, L. Basile, T. Miller, and T.-C. Chiang, Phys. Rev. Lett. 93, 216804 (2004).

[18] L. Basile, H. W. Hong, P. Czoschke, and T.-C. Chiang, Appl. Phys. Lett. 84, 4995 (2004).

[19] Calculations employed the full-potential linearized augmented plane-wave method as implemented in the FLEUR code (www.flapw.de).

[20] M. Grioni, Ch. R. Ast, D. Pacile, M. Papagno, H. Berger, and L. Perfetti, New J. Phys. 7, 106 (2005).

[21] Eli Rotenberg, W. Theis, and K. Horn, J. Alloys Compd. 342, 348 (2002).

[22] S.-J. Tang et al., Phys. Rev. Lett. 96, 216803 (2006). 